\title{
Ajedrez: aportes para personas con Síndrome de Down
}

\section{Chess: contributions for people with Down Syndrome}

\author{
Wilson Hernando Bravo Navarro ${ }^{1}$, Fanny Guadalupe Cadme Galabay ${ }^{1}$ y Santiago Alejandro Jarrin Navas $^{1}$ \\ ${ }^{1}$ Universidad Católica de Cuenca, Ecuador \\ *wilson.bravo@ucacue.edu.ec
}

DOI: https://doi.org/10.26871/killkanasocial.v4i2.614

\begin{abstract}
Resumen
El artículo presenta fundamentalmente el conocimiento de los diferentes aportes de la enseñanza, las terapias lúdicas y artísticas para desarrollar el área cognitiva y socio-afectiva en personas que presentan Síndrome de Down. Se concibe que el Ajedrez, el cual es conocido como el juego ciencia, es una herramienta pedagógica de primer orden que permite el fortalecimiento intelectual, además de ser un agente socializador. Se presenta la propuesta de actividades mediante el juego del Ajedrez con títeres, técnicas plásticas y dramatizaciones como estrategias para viabilizar la inclusión de sujetos con discapacidad intelectual en cualquiera de sus formas. Se parte de la problemática siguiente: las personas con discapacidad intelectual tienen diferentes limitantes, una de las más significativas es la falta de recursos didácticos en cuanto hace referencia al desarrollo mental, el mismo que afecta directamente la esfera social y emociona. De esta manera se procura contribuir en el tratamiento y la eficacia del buen vivir en personas con trastorno genético, en específico con Síndrome de Down, con el empleo del juego como un recurso didáctico, y una alternativa para la reinserción social y una estrategia terapéutica. Se incluyen a varias disciplinas que se adecúan a personas con discapacidad de cualquier índole, por lo que se sugiere un abordaje adaptativo a las necesidades que presentan los usuarios y se brinden diferentes recursos que faciliten el desarrollo de proceso de enseñanza-aprendizaje. Finalmente, luego de haber consultado la bibliografía especializada, se infiere que el uso de las terapias lúdicas es substancial en el proceso enseñanza-aprendizaje de individuos con discapacidad cognitiva.
\end{abstract}

Palabras clave: Ajedrez, herramienta pedagógica, Síndrome de Down, terapias lúdicas.

\begin{abstract}
The article focuses on the knowledge of the different teaching, ludic and artistic therapies' contributions to the development of the cognitive and socio-affective area in people with Down's syndrome. Chess, known as the game of science, is conceived as a first-class pedagogical tool that allows intellectual strengthening, besides being a socializing agent. The proposal is to carry out activities through the Chess game; by using puppets, plastic techniques, and dramatizations, as strategies to facilitate the inclusion of people with intellectual disabilities in any of its forms. The starting point is the following problematic issue: intellectually disabled people have different limitations, being one of the most significant, the lack of educational resources in terms of mental development, which directly affects the social and emotional sphere. This way, it seeks to contribute to the treatment and effectiveness of good living in people with genetic disorders, especially with Down's Syndrome, by using games as an educational resource as well as an alternative therapeutic strategy for social reintegration. Several disciplines, adapted to people with different kinds of disabilities are included; so an adaptive approach to the needs presented by users is suggested, additionally, various resources are provided to facilitate the development of the teaching-learning process. Finally, after having consulted the specialized literature, it is inferred that the use of ludic therapies is essential in the teaching-learning process of individuals with cognitive disabilities.
\end{abstract}

Keywords: Chess, pedagogical tool, Down's Syndrome, ludic therapies.

\section{Introducción}

De acuerdo con estudios realizados a nivel mundial, se develan estadísticas que muestran una cifra que bordea el 1 billón de individuos que tienen algún tipo de imposibilidad, las mismas forman parte del $15 \%$ de la población mundial, las cuales vivencian dificultades para la adaptación a una sociedad que discrimina, violenta y no está preparada para la convivencia con ellas, lo que hace que se evidencie la presencia de un sin número de obstáculo que impiden la participación equitativa y en igualdad de oportunidades en todo orden (Mundial, 2011). Uno de los derechos más vulnerados de estos individuos es el derecho a la educación, el que por más esfuerzos que se hayan realizado por diluir esta brecha no siempre suele hacerse realidad en ningún contexto, esta razón imposibilita la accesibilidad a derechos obligatorios y genera dificultades para alcanzar tanto objetivos primordiales como su potencial, esto disminuye su participación social. En todo el mundo, al menos una 
de cada diez personas está discapacitada debido a discapacidades físicas, mentales o sensoriales (es decir, supera 500 millones de personas a nivel mundial), mientras que al menos uno de cada cuatro se ve afectado negativamente por la presencia de discapacidad. (UN, s.f.). Además, el envejecimiento muy a menudo resulta en limitaciones en la visión, la audición, la memoria o las funciones motoras $\mathrm{y}$, a nivel mundial.

Dentro del Ecuador, uno de cada 550 niños nace con Síndrome Down (Jijón, s.f.), si analizamos la cantidad de niños que nacen anualmente podemos determinar que en el Ecuador nacen alrededor de 402 niños (as), 1 posee trisomía 21, o comúnmente llamado Síndrome de Down, considerando que la edad de la madre al concebir al hijo es uno de los factores preponderantes para adquirir esta variación genética. En adición otro inconveniente es la falta de control prenatal a las madres para el diagnóstico oportuno. Afrontar socialmente esta realidad así como los cambios en las actitudes sociales, han ido conduciendo a la aceptación de esta condición ya que con estudios actuales las vidas para los sujetos con trisomía 21 son más humanas y respetadas desde el punto de vista de las discapacidades intelectuales, esta revisión pretende mejorar su estilo y netamente calidad de vida de estos seres, ya que en nuestro país existe una carencia de centros de formación, sensibilización y apoyo, esta revisión señala como la inclusión de la Actividad Física, la cual conlleva consecuencias positivas en el desarrollo así como la disminución del desarrollo cognitivo, motriz y socio afectivo, permitiéndole tener una mejor calidad de vida, entre otros.

Hay varios tipos de discapacidades que pueden afectar la accesibilidad del Ajedrez, como tal dentro de su desempeño ya que no solo se menciona desde el ámbito motriz. Ya que no hay una sola universalmente clasificación aceptada, una lista indicativa de deficiencias incluye lo siguiente:

a) Discapacidades visuales, como ceguera, baja visión y ceguera al color.

b) Discapacidades motoras o de destreza, incluida la ausencia total de miembros o dedos, y parálisis, falta de multas. Control, inestabilidad o dolor en el uso de los dedos, manos, muñecas o brazos.

c) Discapacidades auditivas, que pueden ir desde la sordera total, donde la persona no puede escuchar nada, hasta leve pérdida de audición donde la persona puede sentir los sonidos y el habla, pero le resulta difícil identificar su contenido.

d) Discapacidades cognitivas, una categoría muy amplia, que incluye aproximadamente dificultades en el desempeño de las tareas mentales que pueden ir desde problemas limitados y enfocados que afectan a una cognitiva muy específica función (por ejemplo, la capacidad de entender las matemáticas), a casos graves (por ejemplo, daño cerebral) donde el individuo es incapaz de cuidar cualquiera de sus actividades cotidianas.

e) Discapacidades del habla, que son bastante raras y algunas veces se combinan con otras discapacidades.
Tiene que se debe tener en cuenta que la deficiencia del habla no está asociada con, y no indica, inteligencia limitada.

f) El analfabetismo, que puede no ser una discapacidad física, pero crea barreras considerables para el aprendizaje.

\section{Desarrollo}

El Ajedrez es un juego con una estructura de reglas compleja y el nivel individual de juego depende de la representación de este sistema de reglas y su anticipación y uso creativo durante el juego. La pregunta, se discute si se puede asumir un cambio del Ajedrez a otras capacidades cognitivas y conocimiento con resultados opuestos; (Saariluoma, 2001).

Uno de los periodistas españoles más reconocidos en el mundo del Ajedrez (García, Prólogo De José, y Marina, s.f.), en su obra Ajedrez y Ciencia, Pasiones Mezcladas; señala a España como el país líder en la aplicación social del juego ciencia, enfocándose en los diferentes grupos sociales y cosechando numerosos beneficios, a este grupo se contemplan personas con autismo, asperger, trastorno de atención e hiperactividad (TDAH) y Síndrome de Down.

Los beneficios de la Actividad Física y el Deporte reconocidos en la población general se extiende ahora a personas con necesidades especiales ya sean personas con trisomía 21, diabetes u obesidad, por ejemplo; en el Síndrome de Down (D.S), la heterogeneidad del síndrome y el daño inducido es incluso importante dependiendo del tipo de trisomía.

Dado el creciente número de publicaciones disponibles sobre Actividad Física y Trisomía 21', se hicieron algunas conclusiones que permitieron mostrar que los individuos con incapacidad puedan acceder a un estatus satisfactorio de actividad motora, así como el desarrollo de la capacidad aeróbica (Rimmer, Heller, Wang, y Valerio, 2004).

Sin embargo, los resultados también muestran que la inactividad física es un síntoma característico de los seres humanos con identificación que viven en instituciones (Heath, s.f.), la inactividad física contra la cual es necesario luchar como para todas las personas.

La inactividad inducida por des acondicionamiento provoca una falta de coincidencia de las principales funciones fisiológicas, y por lo tanto disminución de las capacidades físicas. Esto causa la inactividad y un aislamiento social aún mayor. Además, incluso con entrenamiento físico regular, los individuos con Síndrome de Down exhiben un pico aeróbico bajo, así como sus capacidades y ritmos cardíacos que sus compañeros sin discapacidades. (Bricout y cols., 2008).

Existe cierta evidencia de que los juegos complejos como el Ajedrez podrían tener un impacto positivo en estos procesos, en psicología el juego ciencia se investiga bajo diferentes paradigmas. El Ajedrez es considerado como un modelo para procesos cognitivos y habilidades tales como 
la percepción, manejo de información, atención, memoria, pensamiento lógico y resolución de problemas (Gobet y Simon, s.f.).

El Ajedrez es un recurso que beneficia al desarrollo de la capacidad intelectual en todas las personas en efecto, la literatura muestra que existen terapias lúdicas que se pueden desarrollarse a través del uso de esta disciplina deportiva. Investigaciones llevadas a cabo por (Gonzalez, M, s.f.), plantean de acuerdo a las emociones afectivas (...) tareas como: reacción simbólico-afectiva ante una situación agresiva. Además, mediante la interacción entre dos títeres de animales que se genera un conflicto en el que uno golpea al otro. Se determina el comportamiento ante reacciones del niño y sus posibles desempeños en la situación. Así es como se demuestra que, al incorporar el títere al trabajo, agregamos y consolidamos la afectividad y el desarrollo expresivo a partir del uso de este recurso didáctico como es el títere. El niño deja de avergonzarse de manera natural, y se apropia de sus propias emociones, de tal manera que los expresa, situación antes dificultosa al no poder hacerlo de manera libre.

Acotando, con lo anterior señala que la estrategia lúdica de incorporación de los títeres es un instrumento que permite: Desarrollar actividades de aproximación y aprendizaje de la historia del Ajedrez y la elaboración de otras rutas de mejora como pueden ser títeres sombra. Esto implica que según el autor que de manera que se dé un conocimiento propio de Ajedrez, esto ira incorporando la historia de este, lo cual le permite crear su propia historia, motivándolo a aprender del juego.

Estudiar y progresar las determinantes de la aptitud física en individuos con Síndrome de Down aún se encuentra en su niñez. Siguiendo el enfoque de la Clasificación Internacional del Funcionamiento, la Discapacidad y la Salud, (WHO, 2001) puede verse influido debido al estado de salud, el perfil funcional, la participación en actividades de la vida y los factores contextuales de un niño, tanto en la persona como en el entorno. El Síndrome de Down está asociado con muchas condiciones de salud, pero existe una gran variabilidad en las condiciones que presentan los jóvenes. En un estudio, los problemas médicos no se asociaron con la función entre los jóvenes con Síndrome de Down (Pitetti, Baynard, y Agiovlasitis, 2013). Sin embargo, es razonable suponer que los problemas de salud agudos pueden presentar barreras para la aptitud física para el niño con Síndrome de Down y que, cuando se solucionan eficazmente los problemas médicos, se puede facilitar la aptitud física. Los perfiles funcionales de los jóvenes con trisomía 21 también pueden estar ligados con sus niveles de aptitud física. Los datos cuantitativos y cualitativos sugieren que las habilidades cognitivas, conductuales y motoras inferiores pueden representar barreras para la aptitud física de los jóvenes con el síndrome trisomía 21 (Jobling y Cuskelly, 2006). Los teóricos también argumentan que las personas con esta discapacidad tienen a desarrollar una tendencia a patrones de movimiento más lentos, más seguros y precisos debido a las interacciones neurológicas, ambientales y restricciones asociadas a tareas.

El juego, enfocándose a la terapia lúdica se describe generalmente como espontáneo, propósito y desenvolvimiento de roles, así como el uso del espacio corporal y la fuerza en el entorno espacial y material. Dichas descripciones permiten a los observadores identificar y explicar las desviaciones de los patrones esperados que pueden ser atribuibles a una variedad de factores ambientales en lugar de un estado de desarrollo propio. (Stagnitti y Unsworth, 2000). Algunos estudios han utilizado categorías de juego y sus características de desarrollo definibles para hacer comparaciones entre diferentes grupos de niños, y llegar a las prescripciones de intervención. En particular, la relación entre el juego y el cognitivo y el lenguaje de los niños.

Dentro de la incorporación del juego y el deporte, es importante la optimización de procesos relacionados a la educación netamente con el Ajedrez, haciendo que esta proporcione herramientas para la mejora de habilidades como son, pensamiento crítico, percepción espacial, entre otros. Como resultados del juego y terapias, mediante herramientas como objetos, dibujos que ayudan como instrumentos psicológicos que mejoran su conducta y personalidad, así como rasgos de sociabilización a través de establecimiento de reglas (Gonzalez, M, s.f.).

Varios autores, consideran que la presencia del juego en niños infiere en la habilidad de representatividad del mundo exterior, lo cual los ayuda a distinguir entre la perspectiva real y las características del objeto en este caso la pieza del tablero que ayuda a realizar acciones con él, así como desarrollo de complejas habilidades mentales, en una asunción de roles en el juego. Existen grandes inconvenientes de antecedentes de personas con Síndrome de Down y al practicar el Ajedrez. Ya que no están en un momento de gran actividad ya que el agotamiento es un factor preponderante que al menos se manifiesta en los niños.

Esto entra en el rango de que, en el caso de los niños, estos no logran comprender varias situaciones, por ello es más complejo hacerle frente, debido a esto existe una baja receptibilidad a las actividades recreativas. Para ello es importante considerar edad del niño, tipo de niño y la gravedad del estado, así como sus características personales. La edad del niño logra determinar actividades recreativas como son del rango de 0 a 18 años. Denominando esto como pediatría.

Las diferencias entre los niños de estas edades pueden ser abismales, y nada tienen que ver las necesidades de uno de 2 años con las de uno de 6 u otro de 13. Por tanto, las actividades que llevemos a cabo para la satisfacción de dichas necesidades tampoco pueden ser las mismas. En este caso, han de programarse las actividades recreativas por grupos de edad, de modo que las orientadas a niños de más corta edad sean más sencillas e infantiles, y las que se dirijan a edades más avanzadas adquieran una orientación de mayor madurez y complejidad. Esto será garantía de 
interés en el niño y, por tanto, de participación en las actividades.

El juego del Ajedrez en la educación es importante, contemplando beneficios y mejora de actividades sociales, mejorando valores e interacción individual y colectiva. El Ajedrez, es en definitiva una herramienta pedagógica para el desarrollo individual y cognitivo de personas acoplándolos en varios ámbitos, destacando el social.

Hay pocos estudios cuantitativos que determinen si existe una relación causal entre jugar al Ajedrez y tener un coeficiente intelectual alto desde que implantó el programa de Ajedrez en el aula que corroboran que los alumnos que lo usan como herramienta educativa mejoran en matemáticas $\mathrm{y}$, a nivel lingüístico, en aspectos como la lectura.

El método para enseñar a jugar Ajedrez a niños a partir de los 3 años, diseñado por una ajedrecista mundialmente reconocida. El método incluye la entrega de distintos formatos y tamaños del tablero de Ajedrez, relojes, CD musical, títeres representativos de las piezas, un libro sobre el Ajedrez como herramienta pedagógica, libro del alumno, un programa de capacitación para las educadoras, así como seguimiento y asistencia pedagógica durante todo el curso.
En el juego del Ajedrez respeto de normas y reglas acordadas y, en relación con el Núcleo de Identidad y Autonomía, además dentro de la planificación y la puesta en marcha de acción memorista para aprender y pensar secuencias para el juego, este enfoca su atención en el mismo, haciendo esto complicado y dando como opción la búsqueda de opciones diferentes a las aprendidas, tomando conciencia de autonomía del niño en circunstancias de juego. (Ministerio de Educación, 2019).

Por ello dentro de la revisión de aplicación de Ajedrez en personas con Síndrome de Down, menciona que México es pionero en incluir la enseñanza del Ajedrez para personas con síndrome de Down obteniendo numerosos réditos, mejorando habilidades del pensamiento para aplicarlos en la vida, el proyecto fue realizado por integrantes de la Escuela Nacional de Ajedrez de México. Esta escuela dirigida por tres hermanos de apellido Ferriz, el personal docente de mencionada institución utiliza la misma metodología que con los niños que no poseen esta discapacidad intelectual.

Propuesta de plan de acción para el desarrollo cognitivo y socio-afectivo en las personas con Síndrome de Down.

\begin{tabular}{|c|c|c|c|}
\hline $\begin{array}{l}\text { Estrategias } \\
\text { Pedagógicas }\end{array}$ & Objetivos & Actividades & Recursos \\
\hline $\begin{array}{l}\text { Acciones de } \\
\text { percepción }\end{array}$ & $\begin{array}{l}\text { Realizar acciones de } \\
\text { percepción para desarrollar el } \\
\text { conocimiento de las piezas del } \\
\text { juego de Ajedrez. }\end{array}$ & $\begin{array}{l}\text { Motivación } \\
\text { Realizar ejercicios corporales y de } \\
\text { manipulación. } \\
\text { Construcción } \\
\text { Presentar los títeres representados } \\
\text { con las piezas de Ajedrez. } \\
\text { Jugar con los títeres realizando } \\
\text { movimiento para ejercitar al títere. } \\
\text { Realizar ejercicios de movimiento } \\
\text { del cuerpo. } \\
\text { Crear ejercicios gestuales. }\end{array}$ & $\begin{array}{l}\text { Tablero de Ajedrez con } \\
\text { dimensiones de } 25 \text { metros } \\
\text { cuadrados. } \\
\text { Títeres con las piezas de } \\
\text { Ajedrez. }\end{array}$ \\
\hline $\begin{array}{l}\text { Comunicación } \\
\text { comprensiva }\end{array}$ & $\begin{array}{l}\text { Ejercitar la comunicación para } \\
\text { desarrollar la comprensión del } \\
\text { juego del Ajedrez con teatro } \\
\text { de títeres. }\end{array}$ & $\begin{array}{l}\text { Motivación } \\
\text { Realizar ejercicios de relajación. } \\
\text { Realiza sonidos, gestos y } \\
\text { movimientos con el cuerpo. } \\
\text { Construcción } \\
\text { Manipular y jugar con las piezas } \\
\text { de Ajedrez de forma libre. } \\
\text { Orientar las reglas del juego } \\
\text { presentando las diferentes piezas. } \\
\text { Armar una historia con las piezas } \\
\text { representadas. } \\
\text { Invitar a los autores a expresar sus } \\
\text { acciones de las piezas de Ajedrez. }\end{array}$ & $\begin{array}{l}\text { C.D. } \\
\text { Tablero de ajedrez con } \\
\text { dimensiones de } 25 \text { metros } \\
\text { cuadrados. } \\
\text { Títeres con las piezas de } \\
\text { Ajedrez. } \\
\text { Libreto. } \\
\text { Guión. }\end{array}$ \\
\hline
\end{tabular}




\begin{tabular}{|c|c|c|c|}
\hline $\begin{array}{c}\text { Estrategias } \\
\text { Pedagógicas }\end{array}$ & Objetivos & Actividades & Recursos \\
\hline $\begin{array}{l}\text { Comunicación } \\
\text { expresiva }\end{array}$ & $\begin{array}{l}\text { Representar acciones de } \\
\text { expresión y comunicación } \\
\text { para desarrollar ideas del } \\
\text { juego del Ajedrez con técnicas } \\
\text { plásticas. }\end{array}$ & $\begin{array}{l}\text { Motivación } \\
\text { Presentar los materiales y } \\
\text { herramientas de expresión plástica } \\
\text { Realizar ejercicios de trazos libres } \\
\text { con lápices y crayones. } \\
\text { Construcción } \\
\text { Explicar las técnicas de expresión } \\
\text { plástica } \\
\text { Jugar con pintura de cara para } \\
\text { dibujar de las piezas de Ajedrez. } \\
\text { Orientar las reglas del juego } \\
\text { presentando las diferentes piezas } \\
\text { Armar una escena con las piezas } \\
\text { representadas mediante técnicas } \\
\text { de pintura. } \\
\text { Invitar a los autores a expresar sus } \\
\text { acciones de las piezas de Ajedrez }\end{array}$ & $\begin{array}{l}\text { Lápices de colores } \\
\text { Crayones. } \\
\text { Pintura para cara } \\
\text { Pliegos de cartulina }\end{array}$ \\
\hline
\end{tabular}

De esta revisión se destaca la importancia de las terapias lúdicas dentro del contexto de la actividad física en el ámbito de individuos con Síndrome de Down, ya que es significativo el mantenimiento del pico aeróbico así como una alta actividad motora, lo que permite al infante un mejor desarrollo tanto cognitivo como afectivo, teniendo en cuenta que una de las principales actividades es el Ajedrez como terapia de socialización, inclusión y mejora intelectual de estas personas, así como en una posible extensión del juego de las 64 casillas a diferentes poblaciones donde exista alta incidencia de esta discapacidad intelectual, tomando en cuenta el modelo aplicado en México por docentes integrantes de la Escuela Nacional de Ajedrez, dentro de la viabilidad del proyecto se tiene a España como un referente dentro de la incorporación del Ajedrez social y terapéutico. Cabe destacar que es necesaria la esquematización del deporte progresivo dentro de este contexto, debido a los principios de la Actividad Física, en un aumento gradual de las cargas para llevar un mejor proceso pedagógico.

Es importante articular actividades y acciones dentro del proceso educativo, para personas con Síndrome de Down, pues a partir de las diferencias individuales que confluyen se puede abordar nuevas ideas de enseñanza para poder lograr un cambio importante dentro de la diversidad señalada que, a partir de las concepciones de organización, de las sesiones como estrategia de trabajo.

\section{Referencias Bibliográficas}

Bricout, V. A., Guinot, M., Faure, P., Flore, P., Eberhard, Y., Garnier, P., y Favre Juvin, A. (2008). Are hormonal responses to exercise in young men with Down's syndrome related to reduced endurance performance? Journal of Neuroendocrinology, 20(5), 558-565. doi: 10.1111/j.1365-2826.2008.01695.x

García, L., Prólogo De José, B., y Marina, A. (s.f.). Ajedrez y ciencia, pasiones mezcladas.
Gobet, F., y Simon, H. A. (s.f.). The Roles of Recognition Processes and Look-Ahead Search in TimeConstrained Expert Problem Solving: Evidence from Grandmaster Level Chess (Inf. Téc.).

Gonzalez, M, S. T. (s.f.). PsycNET Record Display PsycNET.

Heath, P. (s.f.). Public Health Perspectives on Disability: Epidemiology to Ethics and Beyond - Google Libros.

Jijón, M. (s.f.). En Ecuador existen 7.457 personas con Síndrome de Down : País : La Hora Noticias de Ecuador, sus provincias y el mundo.

Jobling, A., y Cuskelly, M. (2006, dec). Young people with Down syndrome: A preliminary investigation of health knowledge and associated behaviours. Journal of Intellectual and Developmental Disability, 31(4), 210-218. doi: 10.1080/13668250600999186

Mundial. (2011). Informe mundial de la discapacidad (Inf. Téc.).

Pitetti, K., Baynard, T., y Agiovlasitis, S. (2013). Children and adolescents with Down syndrome, physical fitness and physical activity. Journal of Sport and Health Science, 2(1), 47-57. doi: 10.1016/j.jshs.2012 .10 .004

Rimmer, J. H., Heller, T., Wang, E., y Valerio, I. (2004, mar). Improvements in Physical Fitness in Adults with Down Syndrome. American Journal on Mental Retardation, 109(2), 165-174+195. doi: 10.1352/ 0895-8017(2004)109<165:IIPFIA > 2.0.CO;2

Saariluoma, P. (2001). Chess and content-oriented psychology of thinking (Vol. 22; Inf. Téc.).

Stagnitti, K., y Unsworth, C. (2000). The Importance of Pretend Play in Child Development: An Occupational Therapy Perspective Imagine Create Belong: Supporting the development of social skills for adolescents with social difficulties View project Developing and testing a Mobility Management Pro- 
gram for older drivers View project. British Journal of Occupational Therapy, 63(3). doi: 10.1177/ 030802260006300306

UN. (s.f.). World Programme of Action Concerning Disabled Persons I United Nations Enable.
WHO. (2001). International Classification of Functioning, Disability and Health World Health Organization Geneva ICF ii WHO Library Cataloguingin-Publication Data International classification of functioning, disability and health : ICF (Inf. Téc.).

Recibido: 3 de septiembre de 2019

Aceptado: 20 de mayo de 2020 